\section{Depressão e envelhecimento: estudo nos participantes do Programa Universidade Aberta à Terceira Idade}

\section{Depression and aging: study comprising participants of the "Senior Citizens Open University"}

Valéria Moura Moreira Leite 1 Eduardo Maia Freese de Carvalho 2 Kátia Magdala Lima Barreto 3 Ilka Veras Falcão 4

\begin{abstract}
Objectives: to identify depression in the elderly enrolled at the Senior Citizens Open University, a program of the Federal University of Pernambuco, considering demographic, social and economic variables.

Methods: epidemiological, descriptive, cross sectional cohort study. A census comprising 358 elderly subjects being 312 women and 46 men $(\geq 60$ yr) through the Brasil Old Age Schedule (BOAS) was completed from which the section related to mental health was selected focusing on depression variables such as loneliness, sadness, listlessness, pessimistic view of the future, irritation, self-accusation, suicidal thoughts, headaches, dissatisfaction, sleep and appetite disorders.

Results: an important depression percentage was determined (24.02\%) in the population under study, the majority of which, between 70-79 years old, female and single. A significant association between low education level and depression was established. Depression cases had relevant connection with distress, headache, listlessness, irritation, sadness and dissatisfaction.

Conclusions: depression among the subjects of the study demonstrates the need for the Program to consider actions directed towards participants' health, particularly depression related mental disorders.
\end{abstract}

Key words Depression, Aged, Epidemiology
1,3,4 Departamento de Terapia Ocupacional. Universidade Federal de Pernambuco. Av. Prof. Moraes Rego, 1235. Cidade Universitária. Recife, PE, Brasil. CEP: 50.670-420. E-mail: vmmleite@hotmail.com

2 Departamento de Estudos em Saúde Coletiva. Centro de Pesquisa Aggeu Magalhães. Departamento de Medicina Social da Universidade Federal de Pernambuco, Recife, PE, Brasil

\section{Resumo}

Objetivos: identificar a presença de depressão em idosos que freqüentaram o Programa Universidade Aberta à Terceira Idade, da Universidade Federal de Pernambuco, considerando variáveis demográficas $e$ socioeconômicas.

Métodos: estudo epidemiológico, descritivo e de corte transversal. Foi realizado um censo com os 358 idosos sendo 312 mulheres e 46 homens ( $\geq 60$ anos), utilizando o questionário "Brasil Old Age Schedule" (BOAS), do qual foi selecionada a seção de saúde mental, quanto à depressão, verificando a freqüência das variáveis solidão, tristeza, pouca disposição, pessimismo em relação ao futuro, irritação, auto-acusação, idéias suicidas, dor de cabeça, insatisfação, distúrbios do sono e do apetite.

Resultados: foi encontrado um percentual importante de depressão $(24,02 \%)$ na população estudada, cuja maioria está classificada em depressão menor, entre a faixa etária de 70-79 anos, do sexo feminino e separados. Foi observada associação significante entre baixa escolaridade e depressão. Os casos de depressão apresentam relevante relação com as variáveis preocupação, dor de cabeça, pouca disposição, irritação, tristeza e insatisfação.

Conclusões: a presença de depressão na população estudada aponta para a importância do planejamento, por parte do Programa, de ações direcionadas à saúde de seus participantes, em particular, os transtornos mentais relativos à depressão.

Palavras-chave Depressão, Idoso, Epidemiologia 


\section{Introdução}

O processo de envelhecimento populacional em curso no país tem aumentado a freqüência de doenças psiquiátricas, entre as quais, a depressão, que é a desordem mais comum nesse segmento etário. ${ }^{1,2}$ As taxas de prevalência variam entre $5 \%$ e $35 \%$, quando consideramos as diferentes formas e a gravidade da depressão. ${ }^{3}$

Atualmente, para a identificação de depressão, de acordo com referências mais utilizadas internacionalmente, encontramos os critérios de diagnóstico do "Diagnostic and statical manual of mental disorders",4 que classificam, em escala de sintomas, os casos de depressão em maior ou menor. Os casos de depressão menor são os mais prevalentes na população idosa. 5 Dessa forma, é prudente tomarmos como referência uma abordagem funcional para a depressão no idoso. Quando essa se torna suficientemente severa, a ponto de ser identificada como um caso que demanda atenção clínica, temos certeza de que ocorre certo grau de prejuízo na função. $6 \mathrm{Um}$ aspecto relevante é que, apesar de boa parte das depressões nos idosos apresentar um quadro clínico semelhante ao de outras faixas etárias, esses indivíduos, com maior freqüência, apresentam quadros atípicos, ou particularidades, como problemas clínicos e sociais simultâneos, que podem levar a dificuldades diagnósticas. ${ }^{7}$

Na população envelhecida, a depressão encontrase entre as doenças crônicas mais freqüentes que elevam a probabilidade de desenvolver incapacidade funcional, 8 desencadeando um importante problema de saúde pública, na medida em que inclui tanto a incapacidade individual como problemas familiares em decorrência da doença. Tais fatos somam-se aos custos financeiros, à alta taxa de utilização de serviços de saúde e à diminuição da qualidade de vida. 2,9

Estudos recentes identificam, entre as principais características associadas à depressão, variáveis demográficas, tais como idade avançada e ser do sexo feminino, 5,10 condições de saúde, como o declínio do estado funcional, doenças crônicas e prejuízo cognitivo, ${ }^{8}$ além das condições sociais precárias. ${ }^{2,11}$

No contexto da atual edição da Política Nacional de Saúde do Idoso, e o veloz processo de envelhecimento que ocorre no Brasil, os esforços tendem a concentrar-se em manter o idoso na comunidade, com apoio social junto à sua família, da forma mais digna e confortável possível.12

Nessa perspectiva, foi estudada a depressão nos idosos que fazem parte do Programa Universidade
Aberta à Terceira Idade, da Universidade Federal de Pernambuco (UnATI/UFPE).

O Programa foi criado em setembro de 1996. É uma microuniversidade temática que trata dos aspectos do envelhecimento, a partir de 60 anos, através de atividades de ensino, pesquisa e extensão. Tem como diretriz principal promover a integração Universidade-idoso-comunidade, participando desse processo a comunidade universitária e idosos da comunidade em geral. 13

Portanto, é objetivo deste estudo identificar, dentre os participantes do Programa UnATI/UFPE, a freqüência de depressão, relacionando-a com as variáveis demográficas e socioeconômicas.

\section{Métodos}

Este é um estudo epidemiológico, descritivo e de corte transversal. Sua população é composta por 358 idosos (312 mulheres e 46 homens), procedentes dos vários bairros do Recife e Região Metropolitana, Pernambuco, Brasil que participam do Programa UnATI/UFPE.

Foi realizado um censo desses idosos, a partir de um questionário aplicado em entrevista direta com os participantes do Programa, utilizando o instrumento multidimensional, o "Brazil Old Age Schedule" (BOAS), que foi validado no Brasil, através de pesquisa realizada por Veras, 3 com população idosa, no Rio de Janeiro. Esse instrumento é composto por nove seções que têm como objetivo obter informações gerais dos idosos, saúde física, utilização dos serviços médicos e dentários, atividades da vida diária (AVD), recursos sociais, recursos econômicos, rastreamento de saúde mental, necessidades e pro-blemas que os afetam, além de, no final, possibilitar ao entrevistador uma avaliação.

As entrevistas, previamente agendadas, foram realizadas, nas próprias instalações do Programa, por quatro auxiliares de pesquisa que eram monitores do programa, sendo três acadêmicos de Terapia Ocupacional e um de Fisioterapia, com idade variando de 20 a 23 anos, os quais permaneceram do princípio ao fim do estudo. 13

$\mathrm{O}$ treinamento desses auxiliares iniciou-se pela apresentação dos objetivos do trabalho; em seguida entraram em contato com o questionário para que se familiarizassem com o mesmo. Foi experimentado o instrumento através de entrevistas simuladas, a fim de esclarecer dúvidas no seu manuseio, e realizaramse entrevistas em conjunto para comparação de compreensão de respostas. Após as primeiras dezesseis entrevistas foi retomada a avaliação dessa etapa 
Distribuição das variáveis estudadas e os valores atribuídos.

\begin{tabular}{|c|c|c|}
\hline Variáveis & Perguntas & Pontuação \\
\hline Solidão & Sempre se sente solitário & 1 ponto \\
\hline \multicolumn{3}{|l|}{ Tristeza } \\
\hline & Tem se sentido triste ou deprimido no último mês & 1 ponto \\
\hline & Tem se sentido triste ou deprimido por um período maior que poucas horas & 1 ponto \\
\hline & Sente-se mais triste ou deprimido no início do dia & 1 ponto \\
\hline & Sentiu vontade de chorar e chorou no último mês & 1 ponto \\
\hline \multicolumn{3}{|l|}{ Pouca disposição } \\
\hline & Sente que está ficando mais lerdo ou com menos energia & 1 ponto \\
\hline & Sente-se mais lerdo ou com menos energia no período da manhã & 1 ponto \\
\hline & No último mês, tem estado com menos energia que de costume & 1 ponto \\
\hline & No momento, sente falta de energia para fazer suas coisas no seu dia-a-dia & 1 ponto \\
\hline \multicolumn{3}{|c|}{ Pessimismo em relação ao futuro } \\
\hline & Não menciona expectativas para o futuro & 1 ponto \\
\hline & Futuro descrito negativamente & 2 pontos \\
\hline \multirow[t]{2}{*}{ Irritação } & Sentiu-se mais irritado/zangado do que de costume no último mês & 1 ponto \\
\hline & Fica chateado porque não sai de casa sempre que precisa ou quer sair & 1 ponto \\
\hline \multicolumn{3}{|l|}{ Auto-acusação } \\
\hline & Arrepende-se sobre a vida e se culpa sobre isso e não pensa no assunto no momento & 1 ponto \\
\hline & Arrepende-se sobre a vida e se culpa sobre isso e pensa bastante sobre o assunto & 2 pontos \\
\hline \multicolumn{3}{|l|}{ Idéias suicidas } \\
\hline & No último mês, sentiu que viver não valia a pena & 1 ponto \\
\hline & Rejeita suicídio & 1 ponto \\
\hline & Pensamentos suicidas & 2 pontos \\
\hline & Considerou o suicídio & 3 pontos \\
\hline & Tentou o suicídio & 4 pontos \\
\hline Dor de cabeça & Teve dor de cabeça no mês passado & 1 ponto \\
\hline \multicolumn{3}{|l|}{ Insatisfação } \\
\hline & No momento, sente que perdeu o interesse ou a satisfação pelas coisas & 1 ponto \\
\hline & Perda de interesse causada por depressão/nervosismo & 1 ponto \\
\hline & Sente-se infeliz no momento & 1 ponto \\
\hline Distúrbios do sono & Dificuldade para dormir devido à preocupação, ansiedade, depressão & 1 ponto \\
\hline Distúrbios do apetite & Não tem se alimentado bem por estar deprimido/preocupado/nervoso & 1 ponto \\
\hline
\end{tabular}

Fonte: Veras RP. País jovem com cabelos brancos. Rio de Janeiro; 1993.3

e esclarecidas novas dúvidas que surgiram, bem como, a rediscussão da compreensão uniforme das respostas e reafirmação dos padrões a serem seguidos por todos. 13

Em relação à população estudada, inicialmente foram contatados 369 idosos, porém onze foram considerados perdas. Quatro perdas por recusa e sete perdas por não comparecimento do idoso no dia agendado para a entrevista, voltando a não comparecer após novo agendamento. No total, as perdas representaram $2,68 \% .13$

As seções de informações gerais, saúde física, utilização dos serviços médicos e dentários, atividades da vida diária (AVD), recursos sociais e recursos econômicos foram investigadas por Barreto. 13 
No presente artigo artigo, foi aplicada uma das seções referente à Seção de Saúde Mental, do BOAS, na qual é avaliada a deficiência cognitiva e depressão. Para isso, foi utilizada a escala do "ShortCare", desenvolvida para dar ênfase particular a essas duas áreas principais de alteração psiquiátrica na velhice e que permite, na realidade, indicar a suspeita de um "caso". Optou-se por estudar a Seção de Saúde Mental em relação à depressão. Os sinais e sintomas fundamentais para o diagnóstico de depressão são, tristeza, pouca disposição, pessimismo em relação ao futuro, autocrítica, irritação, autoacusação, idéias suicidas, dificuldade de concentração e também distúrbios do sono e do apetite.

Para discriminação de caso e não caso de depressão na escala do "Short-Care", foi utilizada como referência a pontuação validada por Veras ${ }^{3}$, em que o ponto de corte oito e mais foi o que melhor propiciou equilíbrio entre sensibilidade e especificidade para definição de possível caso, chegando ao escore máximo de 28 pontos. Para o caso de depressão menor, foram classificados, nesse grupo, os idosos com ponto de corte de 8 a 12, que apresentaram sintomas depressivos substanciais, podendo ser denominados deprimidos, e, de depressão maior, os idosos que se encontravam no ponto de corte de 13 ou mais e apresentavam distúrbios graves e persistentes, que têm necessidade de assistência profissional de saúde. 3

O banco foi digitado no Programa Epi-info 6.0. Após essa etapa, foi feito o escore de cada questionário dos 358 idosos, a partir da orientação do manual do instrumento, que especifica quais perguntas são consideradas para o escore e o número de pontos atribuídos a cada uma delas (Quadro 1). Só então cada idoso foi classificado nas categorias correspondentes, a saber: sem depressão, depressão menor e depressão maior.

Uma vez identificados os 86 casos de depressão, entre os 358 idosos estudados, trabalhamos por faixa etária em intervalos de 10 anos, agrupando, assim, as idades entre 60-69 anos e 70-79 anos. Essas duas faixas de idade são caracterizadas como idosos jovens e meio-idosos, respectivamente. ${ }^{3}$

Através do "Analysis do Epi-info", pudemos verificar a freqüência das variáveis em estudo para o grupo de casos de depressão e sem depressão. Nos testes estáticos para as variáveis dicotômicas como sexo e faixa etária foi usado o qui-quadrado com a correção de Yates e para a variável escolaridade foi utilizado o Qui-quadrado para tendência linear.

As variáveis aqui estudadas são as inerentes aos participantes, como idade e sexo, estado conjugal; socioeconômicas, como escolaridade e situação econômica atual. As demais variáveis estão contempladas pelas perguntas da escala de avaliação do "Short-Care", que foram trabalhadas no grupo de casos de depressão e sem depressão, em que o pareamento 1 a 1 foi realizado por ordem da lista de idosos dos dois grupos, obedecendo aos critérios de idade e sexo, compondo uma mostra de 86 participantes para cada grupo. Essas variáveis estão especificadas no Quadro 1 e discriminadas de acordo com os pontos atribuídos a cada uma para o escore

\section{Resultados}

A depressão foi diagnosticada em $86(24,02 \%)$ integrantes da UnATI/UFPE (Figura 1). Desses, 65 $(75,58 \%)$ encontram-se no grupo de depressão menor, enquanto $21(24,42 \%)$ compõem o grupo de

\section{Figura 1}

Distribuição da freqüência dos 358 idosos da Universidade Aberta à Terceira Idade da Universidade Federal de Pernambuco, de acordo com a classificação de casos de depressão e sem depressão.

Sem depressão

Casos de depresssão

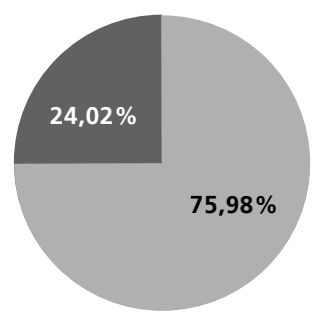

Figura 2

Distribuição da freqüência dos 86 idosos da Universidade Aberta à Terceira Idade da Universidade Federal de Pernambuco, de acordo com a classificação de depressão menor e maior.

Depressão menor

Depressão maior

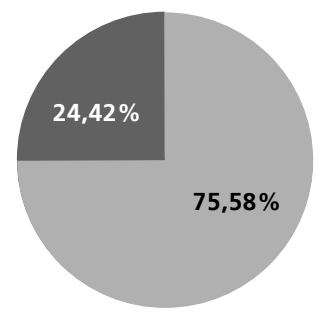


Distribuição dos idosos da Universidade Aberta da Terceira Idade da Universidade Federal de Pernambuco, segundo diagnóstico de depressão e variáveis demográficas e socioeconômicas. Recife, Pernambuco, 1998.

\begin{tabular}{|c|c|c|c|c|c|c|}
\hline & \multicolumn{3}{|c|}{ Com depressão $(n=86)$} & \multicolumn{2}{|c|}{ Sem depressão $(n=272)$} & \multirow[b]{2}{*}{$p$} \\
\hline & $\mathrm{N}$ & $\mathrm{n}$ & $\%$ & $\mathrm{n}$ & $\%$ & \\
\hline \multicolumn{7}{|l|}{ Sexo } \\
\hline Masculino & 46 & 7 & 15,22 & 39 & 84,78 & $p^{*}=0,19$ \\
\hline Feminino & 312 & 79 & 25,32 & 233 & 74,68 & \\
\hline \multicolumn{7}{|l|}{ Faixa etária (em anos) } \\
\hline $60-69$ & 249 & 57 & 22,89 & 192 & 77,11 & $p^{*}=0,36$ \\
\hline $70-79$ & 103 & 29 & 28,16 & 74 & 71,84 & \\
\hline \multicolumn{7}{|l|}{ Escolaridade } \\
\hline Primário & 85 & 27 & 31,76 & 58 & 68,24 & \\
\hline Primeiro grau completo & 47 & 12 & 25,53 & 35 & 74,47 & $p=0,15$ \\
\hline Segundo grau completo & 120 & 28 & 23,33 & 92 & 76,67 & $p^{* *}=0,02$ \\
\hline Curso superior & 103 & 18 & 17,48 & 85 & 82,52 & \\
\hline \multicolumn{7}{|l|}{ Estado conjugal } \\
\hline Nunca casou & 52 & 11 & 21,15 & 41 & 78,85 & \\
\hline Casado & 128 & 26 & 20,31 & 102 & 79,69 & $p=0,47$ \\
\hline Viúvo & 141 & 38 & 26,95 & 103 & 73,05 & \\
\hline Separado & 37 & 11 & 29,73 & 26 & 70,27 & \\
\hline \multicolumn{7}{|c|}{ Situação econômica (atual comparada a quando o entrevistado tinha 50 anos) } \\
\hline Melhor & 146 & 31 & 21,23 & 115 & 78,77 & \\
\hline A mesma & 97 & 24 & 24,74 & 73 & 75,26 & $p=0,55$ \\
\hline Pior & 115 & 31 & 26,96 & 84 & 73,04 & \\
\hline
\end{tabular}

* $\chi^{2}$ Com correção de Yates; **Qui-quadrado de tendência linear

Tabela 2

Distribuição da freqüência das variáveis nos casos de depressão e sem depressão.

\begin{tabular}{|c|c|c|c|c|}
\hline \multirow[t]{2}{*}{ Variáveis } & \multicolumn{2}{|c|}{ Casos de depressão $(n=86)$} & \multicolumn{2}{|c|}{ Sem depressão $(n=272)$} \\
\hline & Freqüência & $\%$ & Freqüência & $\%$ \\
\hline Esteve preocupado durante o último mês & 81 & 94,19 & 57 & 66,28 \\
\hline Preocupa-se em relação a quase tudo & 66 & 81,48 & 31 & 54,39 \\
\hline Dificuldade para dormir devido à preocupação, ansiedade, depressão & 30 & 68,18 & & 50,00 \\
\hline Teve dor de cabeça no mês passado & 52 & 60,47 & 19 & 22,09 \\
\hline Sente que está ficando mais lerdo ou com menos energia & 76 & 88,37 & 47 & 54,65 \\
\hline Sente-se mais lerdo ou com menos energia no período da manhã & 19 & 25,00 & 9 & 19,15 \\
\hline No último mês, tem estado com menos energia que de costume & 34 & 44,74 & 13 & 27,66 \\
\hline No momento, sente falta de energia para fazer suas coisas no seu dia-a-dia & 50 & 65,79 & 14 & 29,79 \\
\hline Sentiu-se mais irritado/zangado do que de costume no último mês & 41 & 47,68 & 15 & 17,44 \\
\hline Tem se sentido triste ou deprimido no último mês & 77 & 89,53 & 22 & 25,58 \\
\hline Sentiu vontade de chorar e chorou no último mês & 55 & 71,43 & 7 & 31,82 \\
\hline No último mês, sentiu que viver não valia a pena & 23 & 26,74 & --- & - \\
\hline No momento, sente que perdeu o interesse ou a satisfação pelas coisas & 41 & 47,67 & 5 & 5,81 \\
\hline Não menciona expectativas em relação ao futuro & 27 & 31,40 & 19 & 22,09 \\
\hline
\end{tabular}


depressão maior (Figura 2).

Na Tabela 1, pode-se observar que, segundo as variáveis demográficas e socioeconômicas, apenas a variável escolaridade foi estatisticamente significante e nas demais houve maior proporção de deprimidos na faixa etária de $70-79$ anos $(28,16 \%)$, no sexo feminino $(25,32 \%)$ e entre os separados $(29,73 \%)$, mas não foram significativas.

A Tabela 2 mostra o resultado das freqüências, das variáveis contempladas pelo "Short-Care", que chamam a atenção no grupo de casos de depressão e sem depressão.

\section{Discussão}

Neste estudo, foram identificados 86 casos de depressão entre os 358 idosos entrevistados. Esse achado em quase um quarto da amostra é considerado elevado em relação aos estudos internacionais realizados em comunidades com idosos, também utilizando escalas para diagnosticar depressão. ${ }^{14}$ Segundo Lima, ${ }^{9}$ essas divergências de percentuais de depressão dos estudos internacionais podem ser atribuídas às diferentes escalas empregadas para diagnosticar depressão, ou a alguns problemas metodológicos, ou, ainda, às possíveis diferenças sócio-demográficas observadas em cada país. 6

$\mathrm{O}$ presente estudo mostra um percentual de depressão nos idosos semelhante aos estudos realizados no Brasil, como o de Veras, ${ }^{3}$ que utilizou a escala da Seção de Saúde Mental do BOAS em condições socioculturais similares. Embora os resultados da maioria dos estudos em relação à alta prevalência de depressão em idade avançada comparada a faixas etárias mais jovens sejam ainda inconclusivos, é importante ressaltar que a depressão é sempre um dos distúrbios psiquiátricos que mais se distinguem na população envelhecida. 3,15

Entre as variáveis demográficas e socioeconômicas encontramos significância estatística de associação da depressão apenas com a variável escolaridade. Contudo, é importante discutir esses resultados quanto à presença de depressão, relacionados com tais variáveis no processo de envelhecimento, à luz de outros estudos similares.

Nos percentuais relativos ao sexo, no grupo de deprimidos, encontramos as mulheres apresentandose em maioria com relação aos homens. Esse achado pode estar relacionado ao que acontece em quase todo o mundo, em que as mulheres vivem, em média, mais do que os homens. 16 Observou-se um maior número de mulheres idosas freqüentando Programas de Universidades Abertas à Terceira Idade, pare- cendo ser essa uma realidade comum entre tais programas no Brasil.17 Comparando com outros estudos de depressão, quanto à predominância de sexo feminino, em idosos na comunidade, observamos semelhanças com os estudos realizados por Cacciatore, 5 na Itália, e com as pesquisas de Veras, 3 no Rio de Janeiro, e Ramos et al., 10 em São Paulo.

As mulheres alcançam idades mais avançadas acompanhadas por uma maior incidência de doenças crônicas, entre elas, a depressão. Isso implica que o aumento da longevidade, principalmente das mulheres, significa aumento da possibilidade de ser acometido por determinada doença crônica. 18,19 Embora neste estudo não tenha sido verificada a relação de saúde física e depressão, Veras, ${ }^{3}$ Stoppe Júnior e Louzã Neto, 7 Penninx et al. 8 apontam que um maior comprometimento da saúde física nas mulheres idosas pode estar relacionado com o aparecimento mais freqüente de depressão nesse grupo.

Quando relacionamos os casos de depressão com faixa etária, encontramos que grande parte dos idosos deprimidos estavam no grupo de idade entre 70-79 anos. Esse resultado pode estar relacionado às descobertas feitas em estudos para diagnosticar depressão em idosos, em que se verificou que a depressão aumentava com a idade.10 Dos idosos identificados como casos de depressão, a maioria apresenta depressão menor. Esses resultados estão em consonância com outros estudos que encontraram uma maior prevalência de depressão menor na população envelhecida. 3,5

A associação encontrada entre escolaridade e depressão, observada na relação de menor escolaridade/maior números de idosos deprimidos, é encontrada em outros estudos, como o de Cacciatore, 5. Entretanto, Black e Markides, 20 em pesquisa populacional com idosos mexicanos, observaram que o grau de escolaridade apresentava fraca associação com depressão.

Em relação à variável que considera a presença de preocupação nos idosos entrevistados, essa foi elevada nos idosos de um modo geral, especialmente no grupo de deprimidos. Possivelmente esse dado se deve ao fato de que, com o passar dos anos, os idosos se tornam mais suscetíveis aos problemas de saúde, dificuldades financeiras, perdas afetivas e sociais, o que aumenta a necessidade de assistência integrada que contemple essa vulnerabilidade. Considerando que no Brasil as condições de assistências à saúde, previdenciárias e sociais são precárias, a maioria dessas responsabilidades são transferidas para o próprio idoso, provocando sentimentos de medo e preocupação. 
Quando consideramos a presença de cefaléia e depressão no processo de envelhecimento, a depressão pode apresentar-se como uma exacerbação dos sintomas somáticos, em que dor de cabeça aparece no conjunto de sintomas clássicos de depressão. ${ }^{21}$ Nesse sentido, a cefaléia pode ser o sintoma principal de um quadro mascarado de depressão. 21 Shuu-Jiun et al.,22 em estudo comunitário, verificaram uma alta co-morbidade entre dor de cabeça e depressão, observando, ainda, que dor de cabeça referida durante o último ano era um im-portante preditor de depressão no idoso. Nessas situações, encontramos suporte na busca de explicações para o grande percentual de idosos deprimidos que, neste estudo, referiram ter tido dor de cabeça.

A depressão pode provocar fadiga persistente, mesmo sem esforço físico, e com ela as atividades mais leves parecem exigir esforço substancial. ${ }^{23} \mathrm{Em}$ consonância com tal afirmação, relativamente aos idosos da UnATI/UFPE, constatou-se um elevado percentual de deprimidos com diminuição da capacidade física para atividades rotineiras.

O humor depressivo é o sintoma mais característico da depressão. 24 Entre os idosos aqui estudados, observamos um alto percentual dos casos de depressão que referem sensação de tristeza. Diferentemente dos nossos resultados, Carvalho e Fernandez 25 referem que os idosos falam muito pouco acerca dos seus sentimentos, comumente negam estar tristes enquanto relatam apatia ou sensação de vazio. Nesse mesmo sentido, em um estudo longitudinal com idosos em uma comunidade, para verificar a presença dos sintomas incluídos nos critérios de diagnóstico para depressão, foi observado que tristeza era o sentimento menos referido pelos idosos. 24 No entanto, para Blazer e Koenig, 6

\section{Referências}

1. Chaimowicz F, Ferreira TJXM, Miguel DFA. Use of psychoactive drugs and falls among older people living in a community in Brazil. Rev Saúde Pública 2000; 34: 631-5.

2. Mann A. Depression in the elderly: findings from a community survey. Maturitas 2001; 38: 53-9.

3. Veras RP. País jovem com cabelos brancos: a saúde do idoso no Brasil. Rio de Janeiro: Relume Dumará; 1994.

4. American Psychiatric Association. Critérios diagnósticos do DSM-IV: referência rápida. Porto Alegre: Artes Médicas; 1995.

5. Cacciatore F. Morbidity patterns in aged population in Southern Italy: a survey sampling. Arch Gerontol Geriatr 1998; 26: 201-13.

6. Blazer DG, Koenig HG. Transtornos do humor. In: Blazer DG, Koenig HG. Psiquiatria geriátrica. Porto Alegre: Artes Médicas; 1999. p. 245-72. as mulheres idosas têm maior chance de admitir e queixar-se de sentimentos depressivos do que os homens idosos, que habitualmente escondem mais seus sentimentos.

Encontramos um elevado percentual de insatisfação nos casos de depressão. A maioria dos idosos deprimidos estudados manifestam perda de interesse ou satisfação pelas coisas. Para Gallo e Rabins, ${ }^{24}$ os idosos deprimidos apresentam freqüentemente uma perda geral de habilidade para sentir prazer, embora a maioria dos estudos longitudinais não tenham verificado forte associação entre depressão e insatisfação na vida. Para Blazer e Koenig, 6 a insatisfação com a vida e a desmoralização podem-se manifestar por auto-estima baixa, desesperança e desamparo, tristeza, pensamento confuso, entre outros motivos, e, mesmo assim, não serem suficientes para preencher o diagnóstico de depressão. Newsom e Schulz, 26 por sua vez, consideram que a escassez de apoio social seja uma importante razão para diminuir a satisfação com a vida na população envelhecida, provocando o aparecimento de depressão.

Contudo, conhecer algumas características da depressão nessa população não significa poder extrapolar os resultados para a população envelhecida em geral, mas pode configurar-se como uma contribuição para melhor conhecer-se a depressão, enquanto um problema epidemiológico relevante, entre os idosos residentes no Recife e área Metropolitana, considerando a abrangência do Programa e a origem dos idosos.

Portanto, a elevada freqüência de depressão na população estudada remete à importância do planejamento, por parte do Programa, de ações direcionadas à saúde dos seus participantes, em particular, os transtornos mentais relativos à depressão.

7. Stoppe Júnior A, Louzã Neto MR. Depressão na terceira idade. São Paulo: Lemos; 1998.

8. Penninx BWJH, Deeg DJH, Eijk JTMV, Beekman ATF, Guralnik JM. Changes in depression and physical decline in older adults: a longitudinal perspective. J Affect Disorders 2000; 61: 1-12.

9. Lima MS. Epidemiologia e impacto social. Rev Bras Psiquiatr 1999; 21: 1-5.

10. Ramos LR, Toniolo NJ, Cendoroglo MS, Garcia JT, Najas MS, Perracini M, Paola CR, Santos FC, Bilton T, Ebel SJ, Macedo MBM, Almada CM, Nasri F, Miranda RD, Gonçalves M, Santos ALP, Fraietta R, Vivacqua I, Alves MLM, Tudisco ES. Two-year follow-up study of elderly: residents in S. Paulo, Brazil: methodology and preliminary results. Rev Saúde Pública 1998; 32: 397-107. 
11. Shoevers RA, Beekman ATF, Deeg DJH, Geerlings MI, Jonker C, Tilburg WV. Risk factors for depression in later life: results of a prospective community based study (AMSTEL). J Affec Disord 2000; 59: 127-37.

12. Negri LSA, Ruy GF, Collodetti JB, Pinto LF, Soranz DR. Aplicação de um instrumento para detecção precoce e previsibilidade de agravos na população idosa. Cienc Saúde Coletiva 2004; 9: 1033-46.

13. Barreto KML, Carvalho EMF, Falcão IV, Lessa FJD, Leite VMM. Perfil sócio-epidemiológico demográfico das mulheres idosas da Universidade Aberta à Terceira Idade no estado de Pernambuco. Rev Bras Saúde Mater Infant 2003; 3: 339-54.

14. Prince MJ, Harwwod RH, Thomas A, Mann AH. Prospective population-based cohort study of the effects of disablement and social milieu on the onset and maintenance of late-life depression. The Gospel Oak Project VII. Psychol Med 1998; 28: 337-50.

15. George LK. Epidemiologia dos transtornos psiquiátricos no idoso. In: Busse EW, Blazer DG. Psiquiatria geriátrica. Porto Alegre: Artmed; 1999. p.167-83.

16. Karsch UM. Idosos dependentes: famílias e cuidadores. Cad Saúde Pública 2003; 19: 861-66.

17. Sant'Anna MJG. UnATI, a velhice que se aprende na escola: um perfil de seus usuários. In: Veras RP, organizador. Terceira idade: desafios para o terceiro milênio. Rio de Janeiro: Relume-Dumará; 1997. p.75-102.

18. Hayflick L. Velhice não é doença. In: Hayflick L. Como e por que envelhecemos. Rio de Janeiro: Campus; 1997. p. $35-41$.

Recebido em 23 de setembro de 2003

Versão final apresentada 29 de novembro de 2005

Aprovado em 17 de janeiro 2006
19. Henderson AS, Jorm LAF, Mackinnon A, Christensen H, Scott LR, Korten AE. The prevalence of depressive disorders and the distribution of depressive symptoms in later life: a survey using draft ICD-10 and DSM-III-R. Psychol Med 1993; 23: 719-29.

20. Black SA, Markides KS. Depressive symptoms and mortality in older Mexican americans. Ann Epidemiol 1998; 9: 45-52.

21. Cunha UGV. Sinais e sintomas em neurologia geriátrica. In: Cunha UGV. Sinais e sintomas em geriatria. Rio de Janeiro: Revinter; 1989. p. 41-57.

22. Shuu-Jiun W, Hsu-Chih L, Jong-Ling F, Chia-Yih L, PeiNing W, Shiang-Ru L. Comorbidity of headaches and depression in the elderly. Pain 1999; 82: 239-43.

23. Del Porto JA. Conceito e diagnóstico. Rev Bras Psiquiatr 1999; 21: 6-11.

24. Gallo JJ, Rabins PV. Depression without sadness: alternative presentations of depression in late life. Am Fam Physician 1999; 60: 820-6.

25. Carvalho VFC, Fernandez MED. Depressão no idoso. In: Papaléo Netto M, organizador. Gerontologia. São Paulo: Atheneu; 1996. p. 160-3.

26. Newsom JT, Schulz R. Social support as a mediator in the relation between functional status and quality of life in older adults. Psychol Aging 1996; 11: 34-4. 